\title{
Performance Analysis of 5G Transmission over Fading Channels with Random IG Distributed LOS Components
}

\author{
Dejan Jaksic, ${ }^{1}$ Risto Bojovic, ${ }^{2}$ Petar Spalevic, ${ }^{1}$ Dusan Stefanovic, ${ }^{3}$ and Slavisa Trajkovic ${ }^{4}$ \\ ${ }^{1}$ Faculty of Technical Science, Singidunum University, Belgrade, Serbia \\ ${ }^{2}$ Faculty of Technical Science, University of Pristina, Kosovska Mitrovica, Serbia \\ ${ }^{3}$ High Technical College, University of Niš, Niš, Serbia \\ ${ }^{4}$ Faculty of Economics, University of Pristina, Kosovska Mitrovica, Serbia
}

Correspondence should be addressed to Petar Spalevic; petarspalevic@yahoo.com

Received 6 January 2017; Accepted 21 May 2017; Published 13 July 2017

Academic Editor: Rausley A. A. De Souza

Copyright (C) 2017 Dejan Jaksic et al. This is an open access article distributed under the Creative Commons Attribution License, which permits unrestricted use, distribution, and reproduction in any medium, provided the original work is properly cited.

\begin{abstract}
Mathematical modelling of the behavior of the radio propagation at mmWave bands is crucial to the development of transmission and reception algorithms of new 5G systems. In this study we will model 5G propagation in nondeterministic line-of-sight (LOS) conditions, when the random nature of LOS component ratio will be observed as Inverse Gamma (IG) distributed process. Closedform expressions will be presented for the probability density function (PDF) and cumulative distribution function (CDF) of such random process. Further, closed-form expressions will be provided for important performance measures such as level crossing rate (LCR) and average fade duration (AFD). Capitalizing on proposed expressions, LCR and AFD will be discussed in the function of transmission parameters.
\end{abstract}

\section{Introduction}

Keeping pace with the insatiable demand of wireless data transmission growth has resulted in occurrence of novel 5G technologies that can offer significant increase in cellular capacity and overcoming of the wireless spectrum shortage $[1,2]$. Consequently, research on modelling channel propagation characteristics for wireless $5 \mathrm{G}$ networks in urban environments at mmWave bands carrier frequencies has been intense recently [3-6].

Stochastic channel models for mmWave communications in both indoor and outdoor environments have been mostly characterized with a Rician distribution in line-of-sight (LOS) environments where a dominant path is present and with Rayleigh distribution for NLOS environment scenarios [7]. In [7], it has been shown that the voltage path amplitudes are following a Rician distribution, with $K$-factor ranging within defined set of values for observed (LOS) and (NLOS) conditions in vertical-to-vertical (V-V) copolarized antenna scenario and corresponding set of values for observed (LOS) and (NLOS) conditions vertical-to-horizontal (V-H) crosspolarized antenna scenario.
However, despite the fact shown in [7] that Rician distribution provides the best fit to the measurement data, results of [7] imply that conventional fading models often fall short in accurately modelling the random fluctuations of $5 \mathrm{G}$ wireless channel signal. In [8], it has been concluded that, for accurate 5G systems channel modelling, proposed model should ensure that the channel LOS and NLOS states, the secondorder statistics of the channel, and the channel realizations should change smoothly in the function of time, antenna position, and/or frequency. Ricean $K$-factor has already been observed as a random variable with determined PDF over the distance in 5G communication in [9]. The Ricean $K$-factor, ratio of powers dominant and scatter components, has been already treated as log-normal random process in [10], but for the narrow-band fixed wireless channels. In [11] it has been shown that log-normal random process, in the mathematical form which is hard for analytically tracking wireless performances, could be efficiently approximated with slowly varying Inverse Gamma (IG) distributed random process.

In this paper we will obtain novel characterization of propagation in LOS conditions, by observing Ricean $K$-factor 
as Inverse Gamma (IG) distributed random process. Standard first-order statistical characterization for this model will be determined; that is, probability density function (PDF) and cumulative distribution function (CDF) of random envelope process will be obtained in closed representation that is convenient to handle both analytically and numerically. Further, important second-order statistical measures as level crossing rate (LCR) and average fade duration (AFD) will be presented in closed form. Capitalizing on their performances of proposed channel will be discussed in function of the system parameters.

\section{System Model}

Rician distributed random process with conditional PDF, conditioned over Ricean $K$-factor, which is observed as random variable, can be expressed as [12]

$$
\begin{aligned}
p_{x \mid K}(x \mid K)= & \frac{2(1+K) x}{\Omega} \exp \left(-K-\frac{(1+K) x^{2}}{\Omega}\right) \\
& \cdot I_{0}\left(2 x \sqrt{\frac{K(K+1)}{\Omega}}\right)
\end{aligned}
$$

where $I_{0}(x)$ denotes the modified Bessel function of the first kind and zero order [12, Eq. 8.445], and $\Omega$ is defined as $\Omega=$ $E\left(x^{2}\right)$, being average signal power.

In $[11,13]$, it has been shown that the PDF of the Inverse Gamma random variable can be expressed as

$$
p_{K}(K)=\frac{\kappa^{c}}{K^{c-1} \Gamma(c)} \exp \left(-\frac{\kappa}{K}\right),
$$

where $\Gamma(x)$ is the Gamma function [12, Eq. 8.310.1], $c>0$ is the shape parameter, and $\kappa>0$ is scale parameter.

Now, novel random process envelope PDF can now be obtained by averaging over IG distributed process of $K$-factor change as

$$
p_{x}(x)=\int_{0}^{\infty} d K p_{x \mid K}(x \mid K) p_{K}(K)
$$

Now after substituting (2) into (3), by performing some mathematical transformations with respect to [12, Equation 3.471.9], we obtain closed-form expression:

$$
\begin{aligned}
& p_{x}(x) \\
& =\sum_{p=0}^{\infty} \sum_{n=0}^{p+1}\left(\begin{array}{c}
p+1 \\
n
\end{array}\right) \frac{x^{2 p+1} \kappa^{c}}{\Omega^{p+1} \Gamma(p+1) \Gamma(c) p !} \exp \left(-\frac{x^{2}}{\Omega}\right) \\
& \quad \times 2\left(\frac{\kappa \Omega}{\Omega+x^{2}}\right)^{(p-n+c) / 2} K_{p-n+c}\left(2 \sqrt{\frac{\kappa\left(\Omega+x^{2}\right)}{\Omega}}\right),
\end{aligned}
$$

where $K_{v}(x)$ denotes the modified Bessel function of the second kind and $v$ th order. Infinite-series from above rapidly converge with only 10-15 terms needed to be summed in each sum in order to achieve accuracy at 5th significant digit.

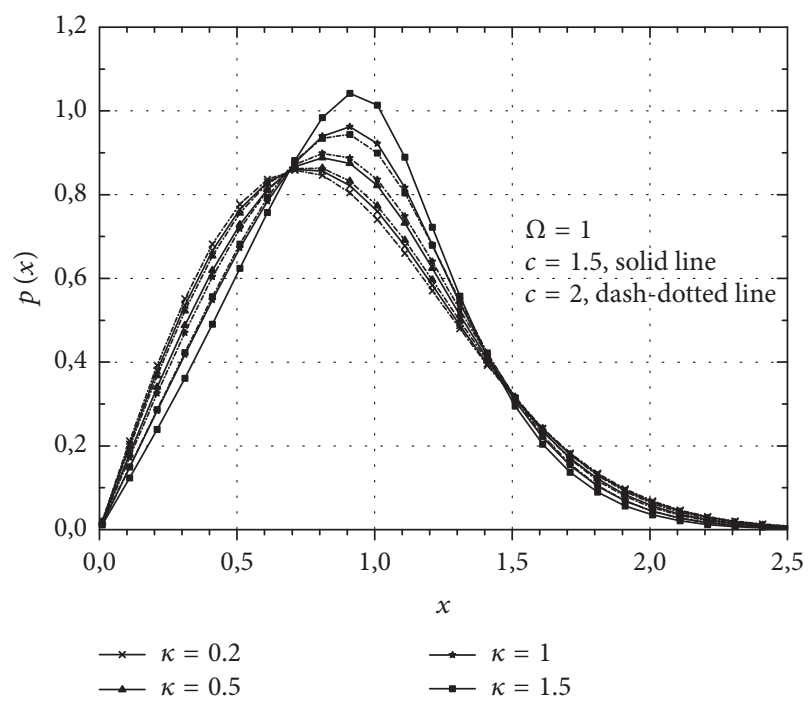

FIGURE 1: PDF of process for various values of system parameters.

In Figure 1, PDF of process is shown for some combination of system parameter values.

Now, by taking into account IG modelled randomness of LOS component, corresponding CDF could be obtained as

$$
\begin{aligned}
F_{x}(x) & =\int_{0}^{\infty} d x p_{x}(x) \\
& =\int_{0}^{\infty} d x \int_{0}^{\infty} d K p_{x \mid K}(x \mid K) p_{K}(K) .
\end{aligned}
$$

After changing integration order, previous relation reduces to

$$
\begin{aligned}
F_{x}(x)= & \int_{0}^{\infty} d K \sum_{p=0}^{\infty} \frac{K^{p} \exp (-K)}{\Gamma(p+1) p !} \gamma\left(p+1, \frac{(1+K) x}{\Omega}\right) \\
& \cdot p_{K}(K),
\end{aligned}
$$

where $\gamma(a, x)$ stands for the incomplete Gamma function [12, Equation 8.443]. Further, with respect to [12, Equation 3.471.9], we can obtain closed-form expression:

$$
\begin{aligned}
& F_{x}(x)=\sum_{p=0}^{\infty} \sum_{n=0}^{\infty} \sum_{s=0}^{p+n+1}\left(\begin{array}{c}
p+n+1 \\
s
\end{array}\right) \\
& \cdot \frac{x^{p+n+1} \kappa^{c}}{\Omega^{p+n+1} \Gamma(p+n+2) \Gamma(c) p !} \exp \left(-\frac{x}{\Omega}\right) \\
& \times 2\left(\frac{\kappa \Omega}{\Omega+x}\right)^{(p+s-c) / 2} K_{p+s-c}\left(2 \sqrt{\frac{\kappa(\Omega+x)}{\Omega}}\right) .
\end{aligned}
$$

Similarly, as in (4), infinite-series from above rapidly converge with only 10-15 terms needed to be summed in each sum in order to achieve accuracy at 5th significant digit.

The average LCR at the determined threshold $x$ is defined as the rate at which the envelope ratio crosses the threshold $x$ 
in a positive or a negative direction and is analytically defined by $[14]$

$$
N_{x}(x)=\int_{0}^{\infty} \dot{x} p_{\dot{x} x}(\dot{x}, x) d \dot{x}
$$

where joint probability density function (JPDF) of the observed random process, $x$, and its derivative with respect to time, $\dot{x}$, denoted by $p_{\dot{x} x}(\dot{x}, x)$, can be evaluated as

$$
p_{\dot{x} x}(\dot{x}, x)=\int_{0}^{\infty} d K p_{\dot{x} x \mid K}(\dot{x}, x \mid K) p_{K}(K),
$$

with $p_{\dot{x} x \mid K}(\dot{x}, x \mid K)$ being JPDF conditioned over random LOS component which is IG distributed.

Conditioned JPDF of the observed random process and its derivative with respect to time can be further presented as

$$
p_{\dot{x} x \mid K}(\dot{x}, x \mid K)=p_{\dot{x} \mid x, K}(\dot{x} \mid x, K) p_{x \mid K}(x \mid K),
$$

where

$$
\begin{gathered}
p_{\dot{x} \mid x, K}(\dot{x} \mid x, K)=\frac{1}{\sqrt{2 \pi} \dot{\sigma}_{x}} \exp \left(-\frac{\dot{x^{2}}}{2 \dot{\sigma}_{x}^{2}}\right) \\
=\frac{1}{\sqrt{2 \pi}\left(\pi f_{d} \sqrt{\Omega /(K+1)}\right)} \\
\cdot \exp \left(-\frac{\dot{x}^{2}}{2 \pi^{2} f_{d}^{2}(\Omega /(K+1))}\right)
\end{gathered}
$$

since $\dot{\sigma}_{x}^{2}=\pi^{2} f_{d}^{2}(\Omega /(K+1))$ for Rician fading channels and $f_{d}$ is the maximum Doppler frequency [15].

After substituting (9), (10), and (11) into (8), expression for average LCR can be presented in the form of

$$
\begin{aligned}
N_{x}(x)= & \int_{0}^{\infty} \dot{x} d \dot{x} \int_{0}^{\infty} d K \\
& \cdot \frac{\exp \left(-\dot{x}^{2} / 2 \pi^{2} f_{d}^{2}(\Omega /(K+1))\right)}{\sqrt{2 \pi}\left(\pi f_{d} \sqrt{\Omega /(K+1)}\right)} \\
& \cdot p_{x \mid K}(x \mid K) p_{K}(K)
\end{aligned}
$$

which after changing order of integration reduces to

$$
\begin{aligned}
& N_{x}(x) \\
& \quad=\int_{0}^{\infty} d K \frac{\left(\pi f_{d} \sqrt{\Omega /(K+1)}\right)}{\sqrt{2 \pi}} p_{x \mid K}(x \mid K) p_{K}(K) .
\end{aligned}
$$

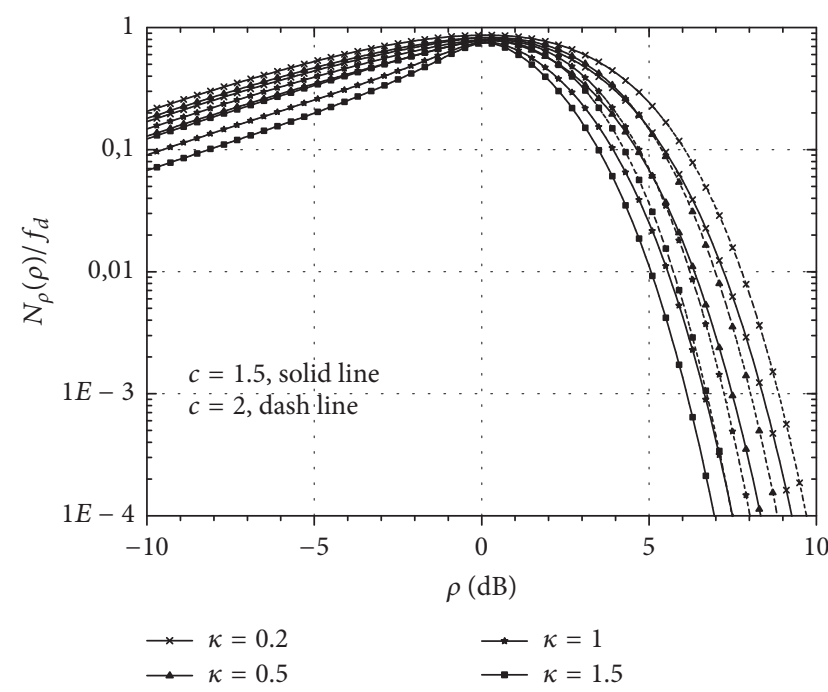

FIGURE 2: Normalized LCR for observed fading channel.

Now by substituting (1) and (2) into (13) with respect to [12, Equation 3.471.9], we can obtain closed-form LCR expression as

$$
\begin{aligned}
& \frac{N_{x}(x)}{f_{d}}=\sum_{p=0}^{\infty} \sum_{n=0}^{p+1}\left(\begin{array}{c}
p+\frac{1}{2} \\
n
\end{array}\right) \\
& \cdot \frac{2 \sqrt{2} \sqrt{\pi} x^{2 p+1} \kappa^{c}}{\Omega^{p+1 / 2} \Gamma(p+1) \Gamma(c) p !} \exp \left(-\frac{x^{2}}{\Omega}\right) \\
& \times\left(\frac{\kappa \Omega}{\Omega+x^{2}}\right)^{(p-n+c) / 2} K_{p-n+c}\left(2 \sqrt{\frac{\kappa\left(\Omega+x^{2}\right)}{\Omega}}\right) .
\end{aligned}
$$

Infinite-series from above rapidly converge with only 10-15 terms needed to be summed in each sum in order to achieve accuracy at 5th significant digit.

\section{Numerical Results}

After introducing $\rho$ as $\rho=x^{2} / \Omega$, LCR expression can be given in the form of

$$
\begin{aligned}
& \frac{N_{\rho}(\rho)}{f_{d}} \\
& \quad=\sum_{p=0}^{\infty} \sum_{n=0}^{p+1}\left(\begin{array}{c}
p+\frac{1}{2} \\
n
\end{array}\right) \frac{2 \sqrt{2} \sqrt{\pi} \rho^{p+1 / 2} \kappa^{c}}{\Gamma(p+1) \Gamma(c) p !} \exp (-\rho) \\
& \quad \times\left(\frac{\kappa}{1+\rho}\right)^{(p-n+c) / 2} K_{p-n+c}(2 \sqrt{\kappa(1+\rho)}) .
\end{aligned}
$$

Figure 2 illustrates the LCR, normalized to $f_{d}$ in the function of parameters $\kappa$ and $c$. As expected, the presence of a stronger LOS component generally causes lower LCR values, while higher values of severity parameters $c$ provide smaller LCR values. 


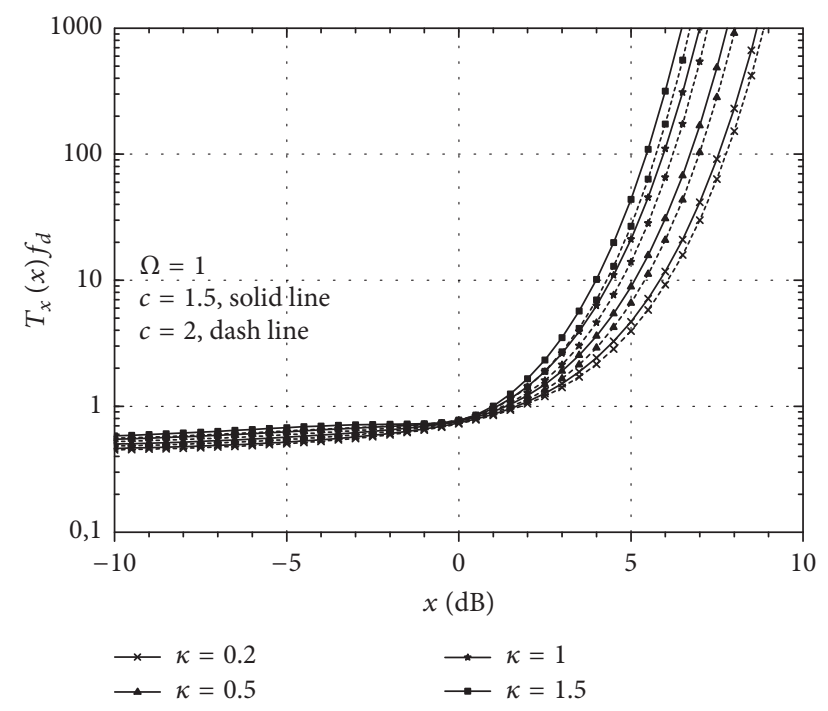

FIGURE 3: Normalized AFD for observed fading channel.

Average time during (AFD) defines time in which the envelope ratio remains below the specified threshold $x$ after crossing that level in a downward direction [14]. AFD can be determined as

$$
T_{x}(x)=\frac{F_{x}(x)}{N_{x}(x)} .
$$

After substituting (14) and (7) into (16) AFD of observed process can be efficiently evaluated. In Figure 3, AFD values are presented, normalized to $f_{d}$ in the function of parameters $\kappa$ and $c$. As expected, higher values of severity parameters $c$ provide smaller AFD values.

\section{Conclusion}

Change of Rician $K$-factor as random IG process in LOS conditioned 5G wireless communications has been considered in this paper, instead of considering Rician $K$-factor as a deterministic variable with constant value defined by 5G communication system properties. Rapidly converging closed-form expressions have been derived for the PDF and CDF of observed propagation signal. Further, based on these expressions, LCR and AFD have been efficiently evaluated and analyzed for observed case in the function of system parameters.

\section{Conflicts of Interest}

The authors declare that there are no conflicts of interest regarding the publication of this paper.

\section{Acknowledgments}

This research was partly funded by Project TR 32023 of Ministry of Science, Serbia.

\section{References}

[1] T. Rappaport, S. Sun, R. Mayzus et al., "Millimeter wave mobile communications for $5 \mathrm{G}$ cellular: it will work!," IEEE Access, vol. 1, pp. 335-349, 2013.

[2] T. S. Rappaport, G. R. MacCartney, M. K. Samimi, and S. Sun, "Wideband millimeter-wave propagation measurements and channel models for future wireless communication system design," IEEE Transactions on Communications, vol. 63, no. 9, pp. 3029-3056, 2015.

[3] M. R. Akdeniz, Y. Liu, M. K. Samimi et al., "Millimeter wave channel modeling and cellular capacity evaluation," IEEE Journal on Selected Areas in Communications, vol. 32, no. 6, pp. 11641179, 2014.

[4] S. Hur, S. Baek, B. Kim et al., "Proposal on millimeter-wave channel modeling for 5G cellular system," IEEE Journal on Selected Topics in Signal Processing, vol. 10, no. 3, pp. 454-469, 2016.

[5] J. Kyrolainen, P. Kyosti, J. Meinila et al., "Channel modelling for the fifth generation mobile communications," in Proceedings of the 8th European Conference on Antennas and Propagation (EuCAP '14), pp. 219-223, IEEE, The Hague, The Netherlands, April 2014.

[6] J. M. Romero-Jerez, F. J. Lopez-Martinez, J. F. Paris, and A. Goldsmith, "The fluctuating two-ray fading model for mmWave communications," in Proceedings of the 2016 IEEE Globecom Workshops (GC Wkshps), pp. 1-6, Washington, DC, USA, December 2016.

[7] M. K. Samimi, G. R. Maccartney, S. Sun, and T. S. Rappaport, " $28 \mathrm{GHz}$ millimeter-wave ultrawideband small-scale fading models in wireless channels," in Proceedings of the 83rd IEEE Vehicular Technology Conference, VTC Spring 2016, May 2016.

[8] K. Haneda, L. Tan, Y. Zheng et al., "5G 3GPP-like channel models for outdoor urban microcellular and macrocellular environments," in Proceedings of the 2016 IEEE 83rd Vehicular Technology Conference (VTC Spring), pp. 1-7, Nanjing, China, May 2016.

[9] A. Rauch, J. Lianghai, A. Klein, and H. D. Schotten, "Fast algorithm for radio propagation modeling in realistic 3-D urban environment," Advances in Radio Science, vol. 13, pp. 169-173, 2015.

[10] L. J. Greenstein, S. S. Ghassemzadeh, V. Erceg, and D. G. Michelson, "Ricean $\mathrm{K}$-factors in narrow-band fixed wireless channels: theory, experiments, and statistical models," IEEE Transactions on Vehicular Technology, vol. 58, no. 8, pp. 40004012, 2009.

[11] S. K. Yoo, P. C. Sofotasios, S. L. Cotton, M. Matthaiou, M. Valkama, and G. K. Karagiannidis, "The Kappa - mu/inverse gamma composite fading model," in Proceedings of the 2015 IEEE 26th Annual International Symposium on Personal, Indoor, and Mobile Radio Communications (PIMRC), pp. 166-170, Hong Kong, China, August 2015.

[12] I. Gradshteyn and I. Ryzhik, Tables of Integrals, Series, and Products, Academic Press, New York, NY, USA, 1980.

[13] V. Witkovský, "Computing the distribution of a linear combination of inverted gamma variables," Kybernetika, vol. 37, no. 1, pp. 79-90, 2001.

[14] X. Dong and N. C. Beaulieu, "Average level crossing rate and average fade duration of selection diversity," IEEE Communications Letters, vol. 5, no. 10, pp. 396-398, 2001.

[15] Z. Hadzi-Velkov, "Level crossing rate and average fade duration of selection diversity with Rician-faded cochannel interferers," IEEE Communications Letters, vol. 10, no. 9, pp. 649-651, 2006. 


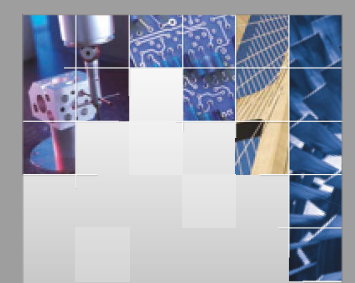

\section{Enfincering}
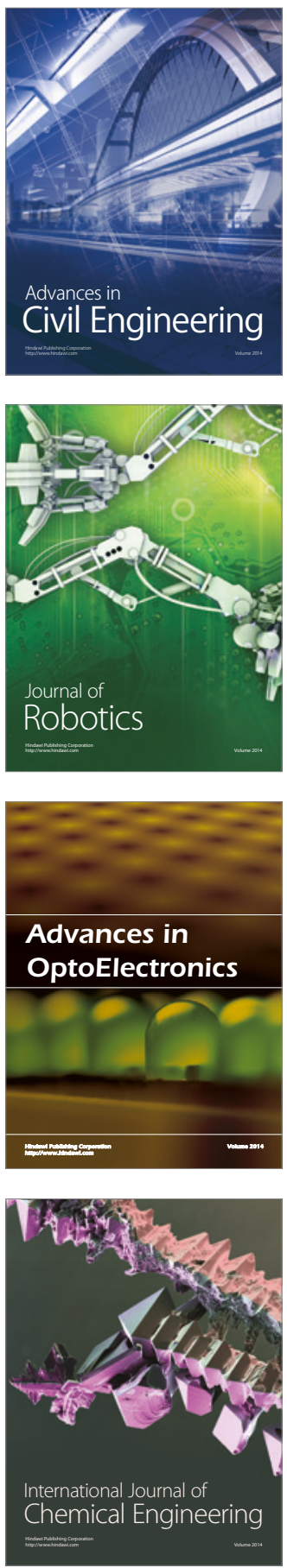

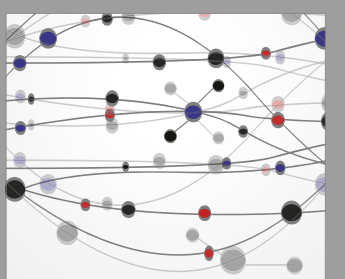

The Scientific World Journal

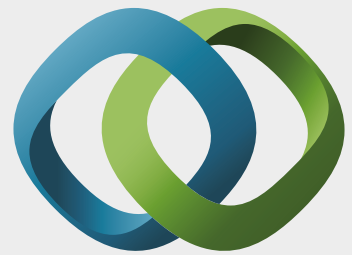

\section{Hindawi}

Submit your manuscripts at

https://www.hindawi.com
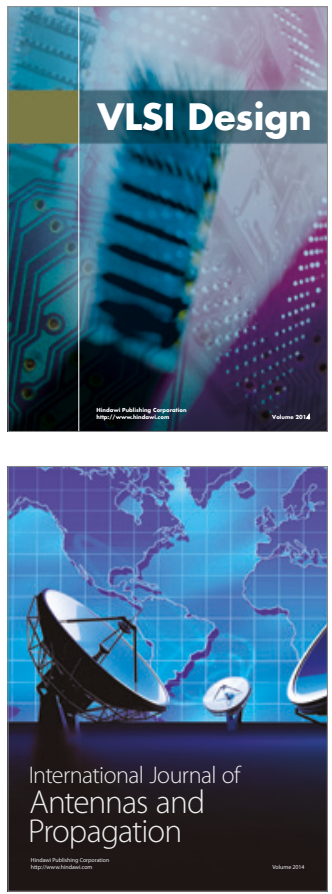

\section{Rotating}

Machinery
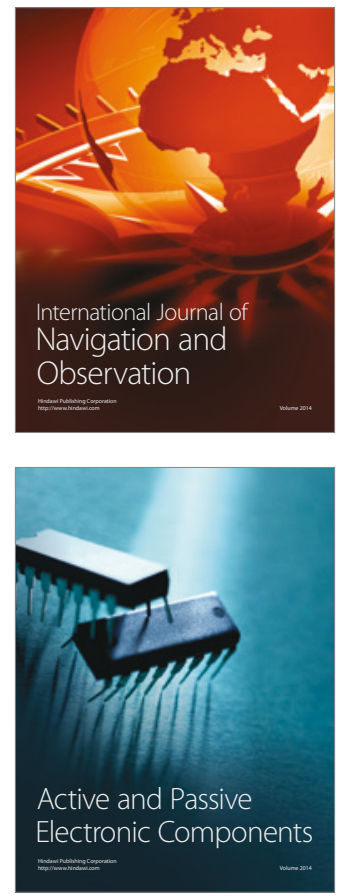
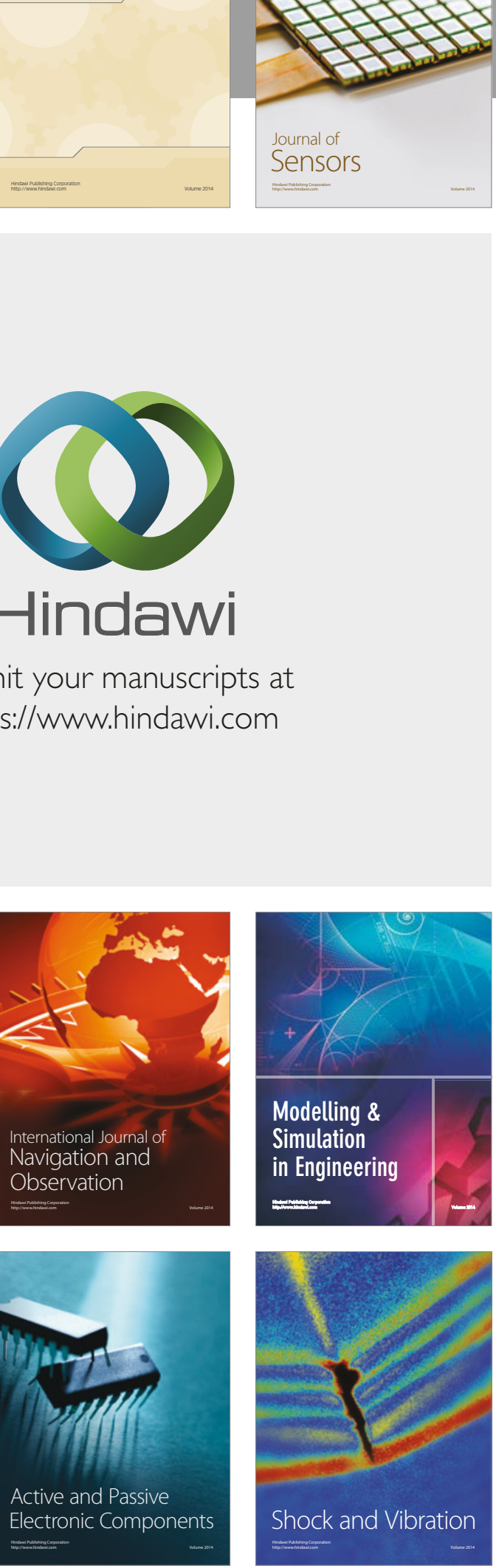
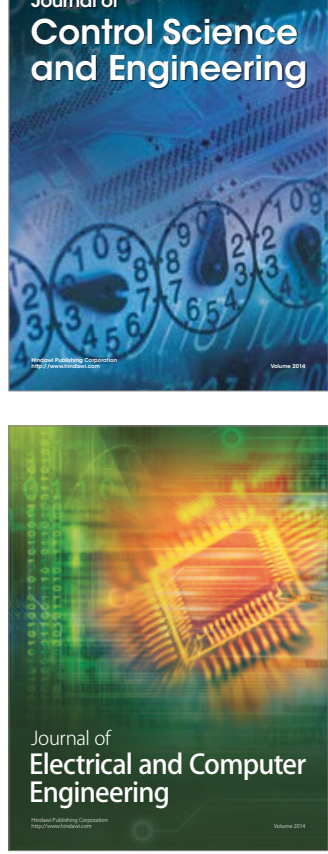

Distributed

Journal of

Control Science

and Engineering
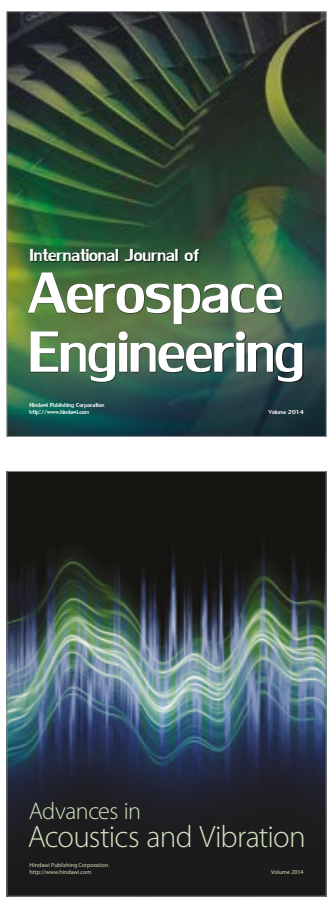

Sensor Networks 